\title{
Numerical Solution of the 2-Hessian Equation by a Newton's Algorithm
}

\section{Haj EA, Khalil $\mathrm{H}$ and Hossein $\mathbf{M}^{*}$}

Laboratory of Mathematics and Its Applications, Lebanese University, Badaro, Beirut, Lebanon

\begin{abstract}
The elliptic 2-Hessian equation is a fully nonlinear partial differential equation that is related, for example, to intrinsic curvature for three dimensional manifolds. We solve numerically this equation with periodic boundary condition and with Dirichlet boundary condition using a Newton's algorithm. We verify numerically, by introducing finite difference schemes, the convergence of the algorithm which is obtained in few iterations.
\end{abstract}

Keywords: Numerical solution; Boundary; Matrix; Periodic function

\section{Introduction}

We are interested by the numerical approximations of the following 2-Hessian, which is a fully nonlinear elliptic partial differential equation in 3-dimensional space $[1,2]$ :

$S_{2}[\psi]=f$ such that $\lambda\left(D^{2} \psi\right) \in \Gamma_{2}$,

Where $S_{2}[\psi]=\sigma_{2}\left(\lambda\left(D_{2} \psi\right)\right)=\sum_{i_{1}<i_{2}} \lambda_{i 1} \lambda_{i 2}=\lambda_{1} \lambda_{2}+\lambda_{1} \lambda_{3}+\lambda_{2} \lambda_{3}$

$\mathrm{D}^{2} \psi=\left(\mathrm{D}_{\mathrm{ij}} \psi\right)_{\mathrm{i}, \mathrm{j}=1,2,3}$ is the Hessian matrix of $\psi, \lambda\left(\mathrm{D}^{2} \psi\right)$ are the eigenvalues of $\mathrm{D}^{2} \psi$ and $\Gamma_{2=}\left\{\lambda \in \mathbb{R}^{3} \mid \lambda_{1+} \lambda_{2+} \lambda_{3>} 0, \sigma_{2}(\lambda)>0\right\}$ [3]. The operator $S_{2}$ is not elliptic unless $f>0$ and $\lambda\left(D^{2} \psi\right) \in \Gamma_{2}[4,5]$.

In the periodic setting, in eqn. (1) reads as follows [2]: given a positive periodic function $f$ on $\mathrm{T}^{3}=\mathbb{R}^{3} / \mathrm{Z}^{3}$, find a periodic function $u$ : $\mathrm{T}^{3}$ $7 \rightarrow \mathbb{R}$ such that

$$
M_{2}[u]=\sigma_{2}\left[\lambda\left(I+D^{2} u\right)\right]=f \text { on } \mathrm{T}^{3}
$$

where $M_{2}$ is the nonlinear differential operator defined by $M_{2}:=u \mapsto$ $\sigma_{2}\left[\lambda\left(I+D^{2} u\right)\right]$. This equation is none other than eqn. (1) with $\psi$ is of the orm $\frac{1}{2}\left|x^{2}\right|+u$ Note that a necessary condition for eqn. (3) to be well posed is that $\int_{\mathbb{T}^{3}} f=1$ [2].

In the next place, we solve numerically the Dirichlet problem

$$
\left\{\begin{array}{l}
S_{2}[\mathrm{u}]=\mathrm{f} \quad \text { in } \Omega=[0,1]^{3} \\
u=0 \quad \text { on } \partial \Omega
\end{array}\right.
$$

which obtained when treating computationally prescribed curvature problems.

\section{Properties of the 2-Hessian Operator}

\section{Proposition 1.1.}

$$
\begin{aligned}
& \text { We have } \\
& S_{2}[u]=u_{x x} u_{y y}+u_{x x} u_{z z}+u_{y y} u_{z z}-u_{x y}^{2}-u_{x z}^{2}-u_{y z}^{2} \\
& M_{2}[u]=3+2 u_{x x}+2 u_{y y}+2 u_{z z}+u_{x x} u_{y y}+u_{x x} u_{z z}+u_{y y} u_{z z}-u_{x y}^{2}-u_{x z}^{2}-u_{y z}^{2}
\end{aligned}
$$

Proof. For a $3 \times 3$ matrix $M$, the characteristic polynomial is given by $\operatorname{det}(M)-c(M) \lambda+\operatorname{trace}(M) \lambda^{2}-\lambda^{3}$

where $c(M)$, the sum of the principal minors of $M$ is given by

$$
\mathrm{c}(M)=\frac{1}{2}\left(\operatorname{trace}(M)^{2}-\operatorname{trace}\left(M^{2}\right)\right) .
$$

Then $c(M)=\lambda_{1} \lambda_{2+} \lambda_{1} \lambda_{3+} \lambda_{2} \lambda_{3}$, where $\lambda_{1}, \lambda_{2}$ and $\lambda_{3}$ are the eigenvalues of $M$. Therefore, by expanding in eqn. (7) and using in eqn. (2), we obtain in eqns. (5 and 6).

\section{Algorithm of Resolution}

Using a global convergence Newton method [1], to linearize the eqn. (1), the algorithm we consider reads: Given $u_{0}$, loop over $n \in \mathrm{N}$,

- Computation of $f_{n=} T_{2}\left[u^{n}\right]$.

- Computation of $\theta^{n}$ as solution of the linearized 2-Hessian equation

$$
\nabla \mathrm{T}_{2}\left[u^{n}\right] \cdot \theta^{n}=\frac{1}{\tau}\left(f-f_{n}\right)
$$

with the stabilization factor $\tau \geq 1$.

- Computation of $u^{n+1=} u^{n+} \theta^{n}$.

Where $T_{2} M_{2}$ for the periodic problem and $T_{2} S_{2}$ for the Dirichlet problem. For $\tau=1$, we obtain the classical Newton's method.

\section{Linearization}

Let $s$ be a parameter in $\mathrm{R}$. We have

$S_{2}[u+s v]=c\left(D^{2} u+s D^{2} v\right)$

$=\frac{1}{2}\left(\operatorname{trace}\left(D^{2} u\right)^{2}-\operatorname{trace}\left(\left(D^{2} v\right)^{2}\right)+s\left(\operatorname{trace} D^{2} u \operatorname{trace}^{2} v-\operatorname{trace}^{2} u D^{2} v\right)+o(s)\right.$

Then

$\nabla S_{2}[u] \cdot v=\operatorname{trace}\left(D^{2} u\right) \operatorname{trace}\left(D^{2} v\right)-\operatorname{trace}\left(D^{2} u D^{2} v\right)$

By expanding in eqn. (9) we obtain the linearization of $S_{2}[u]$ and $M_{2}[u]$. For $u \in C^{2}$ :

$\nabla S_{2}[u] v=u_{x x}\left(v_{y y}+v_{z z}\right)+u_{y y}\left(v_{x x}+v_{z z}\right)+u_{z z}\left(v_{x x}+v_{y y}\right)-2\left(u_{x y} v_{x y}+u_{x z} v_{x z}+u_{y z} v_{y z}\right)$

and

*Corresponding author: Hossein M, Laboratory of Mathematics and Its Applications, Lebanese University, Badaro, Museum, Beirut, Lebanon, Tel: 9611 612830; E-mail: mhdhossein@hotmail.com

Received October 16, 2017; Accepted February 26, 2018; Published March 06 2018

Citation: Haj EA, Khalil H, Hossein M (2018) Numerical Solution of the 2-Hessian Equation by a Newton's Algorithm. J Appl Computat Math 7: 393. doi: 10.4172/21689679.1000393

Copyright: (c) 2018 Haj EA, et al. This is an open-access article distributed under the terms of the Creative Commons Attribution License, which permits unrestricted use, distribution, and reproduction in any medium, provided the original author and source are credited. 
$\nabla M_{2}[u] \quad v=u_{x x}\left(v_{y y}+v_{z z}\right)+u_{y y}\left(v_{x x}+v_{z z}\right)+u_{z z}\left(v_{x x}+v_{y y}\right)-2\left(u_{x y} v_{x y}+u_{x z}\right.$ $\left.v_{x z}+u_{y z} v_{y z}\right)+2 v_{x x}+2 v_{y y}+2 v_{z z}$

\section{Proposition 2.1.}

Let $u \in C^{2} . \nabla S_{2}[u]$ and $\nabla M_{2}[u]$ are elliptic if $u$ is 2-admissible.

Proof. see [3].

\section{Numerical Experiments}

We discretize the problem's domain $[0,1]^{3}$ by dividing the domain into a uniform grid with grid space $h$. We denote by $D_{v 1 v 2} u$ the centered second order finite difference discretization of the operator $u_{v 1 v 2}$ for $v_{1}, v_{2}$ $\in\{x, y, z\}$, and by $D^{2, h} u$ the discretization of the Hessian matrix. That is

$$
D^{2, h} u=\left(\begin{array}{ccc}
D_{x x} u & D_{x y} u & D_{x z} u \\
D_{x y} u & D_{y y} u & D_{y z} u \\
D_{x z} u & D_{y z} u & D_{z z} u
\end{array}\right)
$$

The discretization of the 2-Hessian operators are then given by

$$
S_{2}^{h}[u]=\sigma_{2}\left(\lambda\left(D^{2, h} u\right)\right)=c\left(D^{2, h} u\right)
$$

and

$$
M_{2}^{h}[u]=\sigma_{2}\left(\lambda\left(I+D^{2, h} u\right)\right)=c\left(I+D^{2, h} u\right)
$$

\section{Periodic problem}

The numerical scheme connected to the problem in eqn. (3), obtained by using the global convergence Newton method and the discrete operators described above, is given, in each iteration, by the following linear system of $(m+1)^{3}$ equations with $(m+1)^{3}$ unknowns, where $m+2$ is the number of discretization points given by

$$
\begin{aligned}
& h=\frac{1}{m+1} \\
& D_{x x} u_{i, j, k}^{n} \frac{\theta_{i, j+1, k}^{n}-4 \theta_{i, j, k}^{n}+\theta_{i, j, 1, k}^{n}+\theta_{i, j, k+1}^{n}+\theta_{i, j, k-1}^{n}}{h^{2}}+D_{y y,} u_{i, j, k}^{n} \frac{\theta_{i+1, j, k}^{n}-4 \theta_{i, j, k}^{n}+\theta_{i-1, j, k}^{n}+\theta_{i, j, k+1}^{n}+\theta_{i, j, k-1}^{n}}{h^{2}}+ \\
& D_{z z} u_{i, j, k}^{n} \frac{\theta_{i+1, j, k}^{n}-4 \theta_{i, j, k}^{n}+\theta_{i-1, j, k}^{n}+\theta_{i, j+1, k}^{n}+\theta_{i, j-1, k}^{n}}{h^{2}}-2 D_{x y} u_{i, j, k}^{n} \frac{\theta_{i+1, j+1, k}^{n}+\theta_{i-1, j-1, k}^{n}-\theta_{i-1, j+1, k}^{n}-\theta_{i+1, j+1, k}^{n}}{4 h^{2}} \\
& -2 D_{x=2} u_{i, j, k}^{n} \frac{\theta_{i+1, j, k+1}^{n}+\theta_{i-1, j, k-1}^{n}-\theta_{i-1, j, k+1}^{n}-\theta_{i+1, j, k-1}^{n}}{4 h^{2}}-2 D_{y z} u_{i, j, k}^{n} \frac{\theta_{i, j+1, k+1}^{n}+\theta_{i, j-1, k-1}^{n}-\theta_{i, j-1, k+1}^{n}-\theta_{i, j+1, k-1}^{n}}{4 h^{2}} \\
& +2 \frac{\theta_{i+1, j+1, k}^{n}+\theta_{i-1, j, k}^{n}+\theta_{i, j+1, k}^{n}-6 \theta_{i, j, k}^{n}+\theta_{i, j-1, k}^{n}+\theta_{i, j, k+1}^{n}}{h^{2}}=\frac{1}{\tau}\left(f_{i, j, k}-f_{i, j, k}^{n}\right)
\end{aligned}
$$

$h$ for $i, j, k=0, \ldots, m$ and $f_{i, j, k}^{n}=M_{2}^{h}\left[u^{n}\right]$.

The numerical tests are shown in the following two figures.

In Figure 1, we consider the function $f=1+\sin (2 \pi x) \sin (2 \pi y)$ $\sin (2 \pi z)$ and we solve the problem in the tore $\mathrm{T}^{3}$. Figure 1 shows the convergence of the error $\left\|f-f_{n}\right\|_{L^{2}}$. Different grids are used: $m=15$, 20 and 25. In all the cases, 10 iterations are enough to have an error of order $10^{-12}$. And in all the cases the curves are very close, that means that the convergence of the algorithm is almost independent from the grid space. Then the algorithm is efficient even on very coarse grid.

In Figure 2 shows the convergence of the error $\mathrm{k} u_{e x}-u_{n} \mathrm{k}_{L} 2$ in terms of the number of iterations, where $u_{e x}$ is the solution of the eqn. (3) for $f=M_{2}[u]$ with $u(x, y, z)=0.02 \sin (2 \pi x) \sin (2 \pi y) \sin (2 \pi z)$. Here we fix $m=15$ and $\tau=1$.

\section{Dirichlet problem}

We consider the 2-Hessian equation in $\mathrm{R}^{3}$ with Dirichlet boundary conditions:

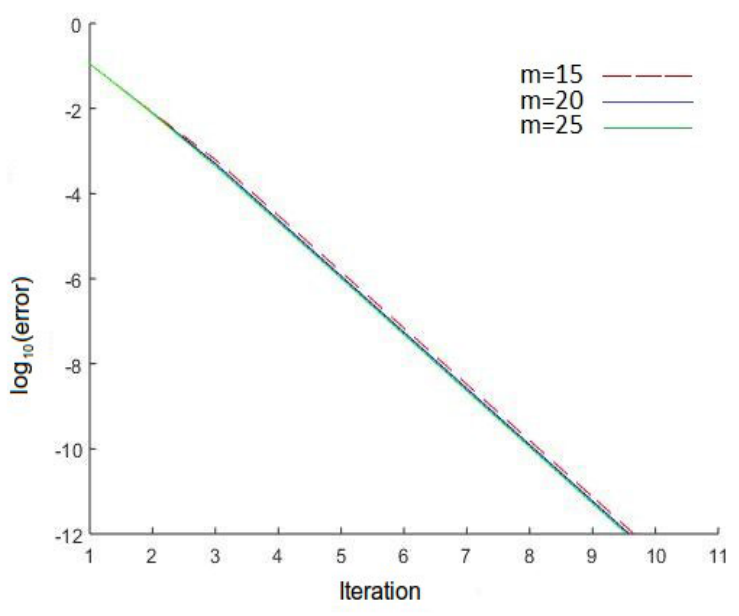

Figure 1: Convergence of the error in norm $L^{2}$ in terms of the number of iterations where the linearized 2 -Hessian equation is solved for the different values of $m: m=25, m=20$ and $m=15$, and for $t=1$.

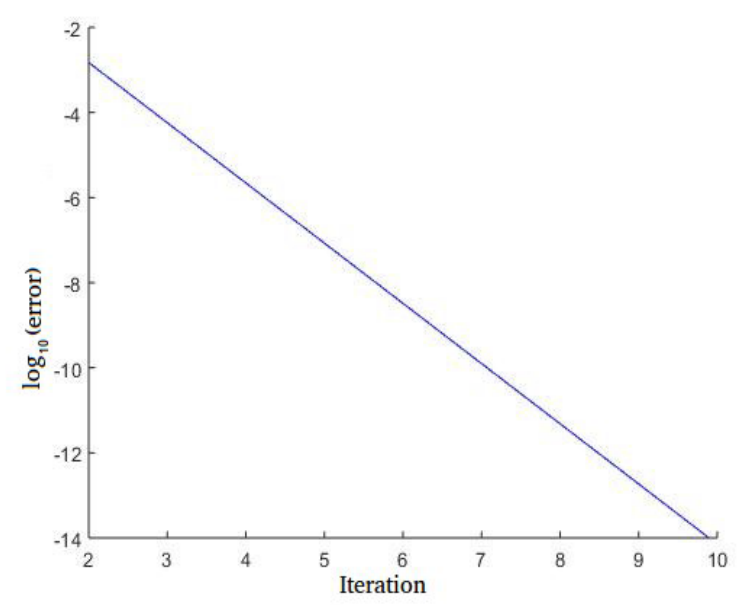

Figure 2: Convergence of the error $k u_{n}-u_{e x} k_{L} 2$ in terms of number of iterations for $m=15$ and $t=1$.

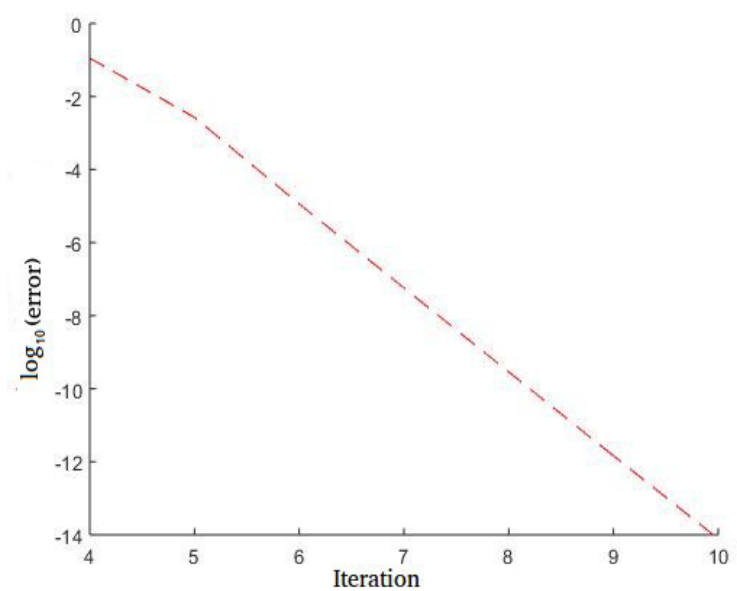

Figure 3: Convergence of the error $\mathrm{k}_{n}-\mathrm{k}_{L} 2$ in terms of number of iterations for $f=4, m=25$ and $t=1$. 


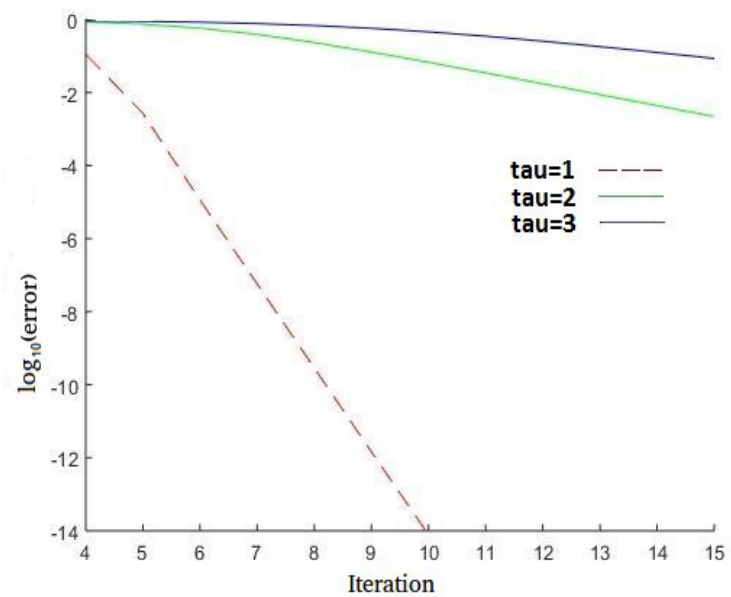

Figure 4: Convergence of the error in norm $L^{2}$ on 15 iterations, for $m=15$ and for different values of $T$.

$$
\left\{\begin{array}{l}
S_{2}[u]=f \text { in } 3=[0,1]^{3} \\
u=0 \quad \text { on } 03
\end{array}\right.
$$

Using the discretization form of $S_{2}[u]$, the numerical scheme connected to the problem in eqn. (12) is given, in each iteration, by the following linear system of $m^{3}$ equations with $m^{3}$ unknowns (Figure 3):

$$
\begin{aligned}
& D_{x x} u_{i, j, k}^{n} \frac{\theta_{i, j+1, k}^{n}-4 \theta_{i, j, k}^{n}+\theta_{i, j-1, k}^{n}+\theta_{i, j, k+1}^{n}+\theta_{i, j, k-1}^{n}}{h^{2}}+D_{y, y} u_{i, j, k}^{n} \frac{\theta_{i+1, j, k}^{n}-4 \theta_{i, j, k}^{n}+\theta_{i-1, j, k}^{n}+\theta_{i, j, k+1}^{n}+\theta_{i, j, k-1}^{n}}{h^{2}}+ \\
& D_{z z} u_{i, j, k}^{n} \frac{\theta_{i+1, j, k}^{n}-4 \theta_{i, j, k}^{n}+\theta_{i-1, j, k}^{n}+\theta_{i, j+1, k}^{n}+\theta_{i, j-1, k}^{n}}{h^{2}}-2 D_{x y} u_{i, j, k}^{n} \frac{\theta_{i+1, j+1, k}^{n}+\theta_{i-1, j-1, k}^{n}-\theta_{i-1, j+1, k}^{n}-\theta_{i+1, j+1, k}^{n}}{4 h^{2}} \\
& -2 D_{x=2} u_{i, j, k}^{n} \frac{\theta_{i+1, j, k+1}^{n}+\theta_{i-1, j, k-1}^{n}-\theta_{i-1, j, k+1}^{n}-\theta_{i+1, j, k-1}^{n}}{4 h^{2}}-2 D_{y=1} u_{i, j, k}^{n} \frac{\theta_{i, j+1, k+1}^{n}+\theta_{i, j-1, k-1}^{n}-\theta_{i, j-1, k+1}^{n}-\theta_{i, j+1, k-1}^{n}}{4 h^{2}} \\
& =\frac{1}{\tau}\left(f_{i, j, k}-f_{i, j, k}^{n}\right)
\end{aligned}
$$

Finally, Figure 4 shows the order of convergence of the algorithm for different values of $\tau$. We remark that the order of convergence decrease very fast in terms of $\tau$. Note that in practice we have taken $\tau=1$, for which value the order of convergence is close to 2 .

\section{Conclusion}

We presented a Newton's algorithm to solve the fully nonlinear 2-Hessian equation in the case where it is elliptic and the solution is smooth enough. The numerical experiments show that the convergence is very fast. Then we can solve the 2-Hessian in the cost of solving a few number of linear elliptic problems. The sparsity of the matrix of discretization allows us to solve quickly the linear problems. Moreover, the numerical tests show a good stability of this algorithm.

\section{References}

1. Bank RE, Rose DJ (1981) Global approximate Newton methods. Numerische Mathematik 37: 279-295.

2. Caffarelli L, Li Y (2004) Un theoreme de Liouville pour les solutions de l'equation de Monge-Ampere avec donnees periodiques. In Annales de I'Institut Henri Poincare/Analyse non lineaire 1: 97-120.

3. Froese BD, Oberman AM, Salvador T (2017) Numerical methods for the 2-Hessian elliptic partial differential equation. IMA Journal of Numerical Analysis 37: 209-236.

4. Loeper G, Rapetti $F$ (2005) Numerical solution of the Monge-Ampère equation by a Newton's algorithm. Comptes Rendus Mathematique 340: 319-324.

5. Hossein M (2009) Solutions entières d'équations hessiennes dans Rn. Comptes Rendus Mathematique 347: 1047-1050. 